\title{
Características sociodemográficas e hábitos de vida de idosos com e sem indicativo de depressão
}

\author{
Sociodemographic characteristics and the lifestyles of aged individuals with or without signs of depression
}

Características sociodemográficas y hábitos de vida de ancianos con y sin signos de depresión

Pollyana Cristina dos Santos Ferreira', Darlene Mara dos Santos Tavares², Nayara Paula Fernandes Martins ${ }^{3}$,

Leiner Resende Rodrigues ${ }^{4}$, Lúcia Aparecida Ferreira ${ }^{5}$

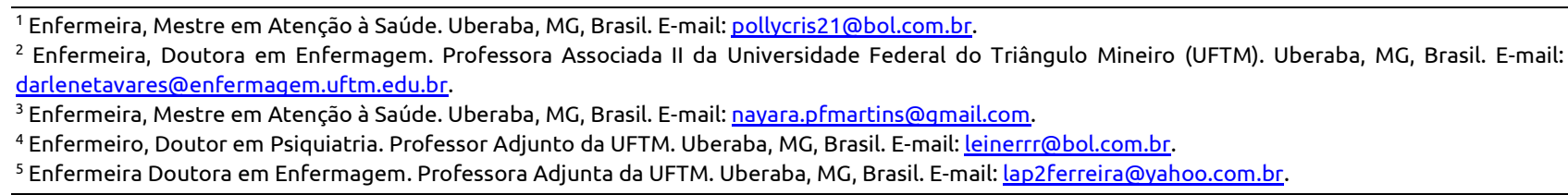

\section{RESUMO}

Objetivou-se comparar as variáveis sociodemográficas e hábitos de vida dos idosos com e sem indicativo de depressão. Trata-se de um inquérito domiciliar, descritivo, transversal e observacional, do qual participaram 850 idosos residentes na zona rural de um município de Minas Gerais. Utilizaram-se os instrumentos estruturados e a Escala de Depressão Geriátrica Abreviada, seguidos de análise descritiva e teste qui-quadrado $(p<0,05)$. Predominaram os idosos com indicativo de depressão do sexo feminino, 80 anos ou mais, donas de casa, que não realizavam atividade física e não faziam uso de álcool, quando comparados aos sem indicativo. A proporção de idosos sem indicativo de depressão foi significativamente superior à dos idosos com indicativo quanto às atividades de passatempo. Não houve diferença significativa entre os grupos em relação ao arranjo de moradia e tabagismo. Conclui-se que esses resultados podem subsidiar o desenvolvimento de ações de prevenção da depressão, além do tratamento e recuperação dos acometidos.

Descritores: Depressão; Enfermagem Geriátrica; Saúde do Idoso.

\section{ABSTRACT}

The objective was to compare the sociodemographic variables and lifestyles of aged individuals with or without signs of depression. This descriptive, cross-sectional and observational home survey, counted with the participation of 850 elderly residents of the rural area of a city in the state of Minas Gerais. Structured instruments and the Abbreviated Geriatric Depression Scale were used, followed by descriptive analysis and chi-square test $(p<0.05)$. Most participants with signs of depression were female, 80 years or older, housewives, engaged in physical activity and did not consume any alcohol, compared with those without signs of depression. The ratio of aged individuals without signs of depression was significantly higher compared to those with signs, regarding the pastime activities. No significant difference was found between the groups regarding housing and smoking. In conclusion, these results could support the development of actions to prevent depression, in addition to the treatment for those with depression.

Descriptors: Depression; Geriatric Nursing; Health of the Elderly.

\section{RESUMEN}

Se objetivó comparar a los ancianos con y sin signos de depresión según variables sociodemográficas y hábitos de vida. Estudio de averiguación domiciliaria, descriptivo, transversal, observacional, con participación de 850 ancianos residentes en zona rural de municipio de Minas Gerais. Se utilizó instrumento estructurado y Escala de Depresión Geriátrica Abreviada; datos evaluados por estadística descriptiva y test Chi-Cuadrado $(p<0,05)$. Predominaron los ancianos con signos de depresión de sexo femenino, 80 años o más, dueñas de casa, sin actividad física, no consumidores de alcohol, en comparación a los que no presentaban signos. La proporción de ancianos sin signos de depresión fue significativamente superior a los que sí los presentaban en cuanto a las actividades recreativas. No existió diferencia significativa entre los grupos en relación a condiciones domiciliarias y tabaquismo. Estos resultados pueden colaborar con el desarrollo de acciones preventivas de la depresión y con el tratamiento y recuperación de los afectados.

Descriptores: Depresión; Enfermería Geriátrica; Salud del Anciano. 


\section{INTRODUÇÃO}

A depressão é um dos transtornos psiquiátricos mais frequentes entre os idosos. Trata-se de uma doença que apresenta multicausalidade, podendo ser desencadeada devido a fatores biológicos, socioeconômicos, psicológicos, culturais, emocionais, entre outros. Os acometidos, quando não diagnosticados e não tratados, podem apresentar comprometimento físico, sociais e funcionais, levando à diminuição da qualidade de vida e, em estágios mais graves, culminando no suicídio. Destacase que as queixas apresentadas pelo idoso devem ser consideradas e investigadas, uma vez que a identificação precoce dos sintomas depressivos, seguido do diagnóstico e início do tratamento é essencial para minimizar os riscos de agravo da doença ${ }^{(1)}$.

Considerada um problema de saúde pública, a depressão requer atenção dos profissionais de saúde, a fim de evitar o sofrimento dos idosos que não recebem tratamento, bem como de seus familiares, e de reduzir os custos financeiros que a doença implica à sociedade e ao poder público(2). Para tanto, é imprescindível que o idoso com depressão seja avaliado considerando os aspectos multidimensionais e especificidades do envelhecimento aliado à complexidade da doença(3).

No Brasil, a prevalência de depressão entre os idosos que residem nas zonas urbana e rural, segundo dados da Pesquisa Nacional por Amostras de Domicílio (PNAD) é de $9,2 \%{ }^{(4)}$. Porém, estudo comparativo realizado com idosos de Quebec ${ }^{(5)}$, no Canadá, verificou maior prevalência de indicativo de depressão entre aqueles que residiam na zona rural (17,0\%), em relação aos da zona urbana $(15,1 \%)$ ou em áreas metropolitanas (10,3\%).

No Brasil, pesquisas realizadas com idosos residentes em zonas rurais destacam que essas localidades apresentam peculiaridades, as quais podem interferir no acesso aos serviços de saúde pelo idoso, como a distância e escassez de transporte ${ }^{(6-7)}$. Adicionalmente, observa-se na zona rural uma realidade em que predominam o baixo nível socioeconômico e de escolaridade, presença de comorbidades crônicas e o isolamento geográfico, culminando na dificuldade de acesso aos recursos sociais e de saúde ${ }^{(8)}$. Esses fatores podem inviabilizar a detecção precoce da depressão e o início e continuidade do tratamento, podendo agravar as condições de saúde do idoso.

Pesquisa realizada com idosos no México, observou a associação entre o indicativo de depressão e residir na zona rural, ter maior idade, presença de comorbidades e de incapacidade funcional e entre aqueles em situação de risco social(9). Em outro inquérito, realizado com idosos na zona rural da China, residir sozinho e não possuir escolaridade esteve associado ao maior risco de apresentar depressão. Além disso, os que nunca tinham fumado ou consumido álcool apresentaram menos sintomas depressivos(10).

Outro fator a ser considerado no desenvolvimento da depressão entre os idosos refere-se às atividades de lazer. Pesquisa conduzida com idosos de um município da Bahia verificou que aqueles inativos no lazer apresentavam maior prevalência de transtornos mentais comuns $^{(11)}$. Ressalta-se que as limitações na zona rural podem restringir as atividades de passatempo e de lazer dos idosos. Estudo com idosos portugueses evidenciou que idosos da zona rural praticavam menos atividade de lazer que aqueles residentes na zona urbana ${ }^{(12)}$. Destacase que as atividades de lazer podem favorecer ao idoso ampliar sua rede de relações interpessoais e a interação com o meio em que vive, contribuindo para o melhor bemestar.

Estudo realizado em Florianópolis-SC verificou que os idosos sedentários em relação à atividade física total, que engloba lazer, ocupação, deslocamentos e serviços domésticos, apresentavam mais chances de apresentar depressão do que aqueles não sedentários ${ }^{(2)}$. Nesse sentido, as melhorias nas condições físicas e na interação social, propiciada pela prática de atividade física, podem minimizar a presença de sintomas emocionais e físicos no idoso(13). Ademais, pode contribuir para a promoção da saúde e prevenção de agravos advindos da depressão.

De acordo com dados do DATASUS ${ }^{(14)}$, em 2010, a população idosa residente na zona rural do Brasil correspondia a 15,9\% e em Minas Gerais a 11,5\%. Sendo assim, faz-se necessária a realização de pesquisas que permitam ampliar o conhecimento sobre as necessidades específicas dessa população e favorecer o desenvolvimento de estratégias de ação para a promoção da saúde e prevenção de doenças no meio rural, além da detecção precoce dos possíveis casos de depressão, para posterior confirmação diagnóstica e estabelecimento do tratamento adequado, contribuindo para melhorias na qualidade de vida desses idosos.

Nessa perspectiva, o presente estudo objetivou comparar os idosos com e sem indicativo de depressão segundo as variáveis sociodemográficas, atividades de passatempo, atividade física, tabagismo e uso de álcool. 


\section{METODOLOGIA}

Esta pesquisa faz parte de um estudo maior desenvolvido pelo Núcleo de Pesquisa em Saúde Coletiva da Universidade Federal do Triângulo Mineiro. Trata-se de um inquérito domiciliar, analítico, transversal e observacional.

A população foi constituída partindo-se de uma listagem de idosos cadastrados na Estratégia de Saúde da Família (ESF) da zona rural, disponibilizada pela Secretaria Municipal de Saúde, totalizando 1.297 pessoas. Destacase que a ESF tem cobertura de $100 \%$ da zona rural do município.

Foram considerados como critério de inclusão para a presente pesquisa: ter 60 anos ou mais de idade, residir na zona rural do município de Uberaba-MG, não apresentar declínio cognitivo e concordar em participar.

Foram excluídos do estudo 447 idosos, dos quais: 117 devido à mudança de endereço, 105 que apresentaram declínio cognitivo, 75 que se recusaram a participar, 57 que não foram encontrados após três tentativas do entrevistador, 11 que haviam ido a óbito, três por estarem hospitalizados e 79 devido a outros motivos. Participaram, assim, 850 idosos; dos quais 187 com indicativo de depressão e 663 sem o referido indicativo.

Os dados foram coletados entre junho de 2010 e março de 2011, por 14 entrevistadores previamente treinados. As entrevistas foram revisadas por supervisores de campo e quando houve questões incompletas ou inconsistentes foram devolvidas aos entrevistadores para preenchimento adequado.

Antes do início da entrevista aplicou-se o Mini Exame do Estado Mental (MEEM), validado no Brasil(15), para verificar se o idoso possuía declínio cognitivo. Este instrumento é composto por questões que abrangem orientação, memória imediata e de evocação, concentração, cálculo, linguagem e domínio espacial, com escore variando de zero a 30 pontos. O ponto de corte é estabelecido de acordo com o a escolaridade do entrevistado, sendo disposto da seguinte maneira: 13 pontos para analfabetos, 18 pontos para aqueles com um a 11 anos de estudo e 26 para acima de 11 anos de escolaridade(15).

Utilizou-se o instrumento semiestruturado baseado no Older Americans Resources and Services e adaptado à realidade brasileira(16) para a coleta das variáveis sociodemográficas, atividades de passatempo, prática de atividade física, tabagismo e uso de álcool. As variáveis estudadas foram: sexo (masculino e feminino); faixa etária (60 -70; 70 -80 e 80 ou mais); arranjo de moradia (vive só; vive somente com cuidador profissional; somente com o cônjuge; com outros de sua geração; com filhos; com netos; outros arranjos); atividade profissional (dona de casa; empregada doméstica; trabalhador braçal; trabalhador rural; profissional liberal; agricultor; empresário; outro e não exerce); atividades de passatempo (assistir televisão; ouvir rádio; ler; jogar; fazer atividades manuais e dançar); satisfação com o lazer (muito insatisfeito; insatisfeito; satisfeito e muito satisfeito); prática de atividade física (sim ou não); tabagista (sim ou não) e frequência que ingere bebida alcoólica (diariamente; uma a três vezes por semana; quatro a seis vezes por semana; uma a três vezes por mês; menos de uma vez por mês; nenhuma).

Aplicou-se a Escala de Depressão Geriátrica Abreviada para avaliar o indicativo ou não de depressão. Utilizou-se a escala adaptada no Brasil( ${ }^{(17)}$. Constituí-se em 15 questões objetivas (sim ou não), com escore que pode variar de zero a 15 pontos. Considerou-se o ponto de corte $5 / 6^{(18)}$.

Os dados foram digitados em dupla entrada no programa Excell ${ }^{\circledR}$ e, posteriormente, foram transportados para o software Statiscal Package for the Social Sciences (SPSS), versão 17.0, para proceder à análise.

Utilizou-se análise descritiva por meio de frequências simples e para a comparação das variáveis aplicou-se o teste qui-quadrado, sendo considerado significativo quando $p<0,05$.

A pesquisa foi aprovada pelo Comitê de Ética em Pesquisa com Seres Humanos da Universidade Federal do Triângulo Mineiro, protocolo No 1477 . Os sujeitos foram contatados nos domicílio, foram prestadas as informações sobre a pesquisa e após a assinatura do Termo de Consentimento Livre e Esclarecido procedeu-se a entrevista.

\section{RESULTADOS}

A Tabela 1, apresenta as características sociodemográficas e econômicas dos idosos, segundo a presença de indicativo de depressão. 
Tabela 1: Características sociodemográficas e econômicas dos idosos com e sem indicativo de depressão. Uberaba, MG, 2011.

\begin{tabular}{|c|c|c|c|c|c|}
\hline \multirow{3}{*}{ Variáveis sociodemográficas e econômicas } & \multicolumn{4}{|c|}{ Depressão } & \multirow{3}{*}{$p$} \\
\hline & \multicolumn{2}{|c|}{ Sim } & \multicolumn{2}{|c|}{ Não } & \\
\hline & $\mathbf{N}$ & $\%$ & $\mathbf{N}$ & $\%$ & \\
\hline \multicolumn{6}{|l|}{ Sexo } \\
\hline Masculino & 68 & 36,4 & 381 & 57,5 & \multirow{2}{*}{$<0,001$} \\
\hline Feminino & 119 & 63,6 & 282 & 42,5 & \\
\hline \multicolumn{6}{|l|}{ Faixa etária } \\
\hline $60+70$ & 110 & 58,8 & 405 & 61,1 & \multirow{3}{*}{0,034} \\
\hline $70+80$ & 52 & 27,8 & 209 & 31,5 & \\
\hline 80 ou mais & 25 & 13,4 & 49 & 7,4 & \\
\hline \multicolumn{6}{|l|}{ Arranjo de moradia } \\
\hline Vive só & 34 & 18,2 & 102 & 15,4 & \multirow{7}{*}{0,573} \\
\hline Somente com cuidador profissional & - & - & - & - & \\
\hline Somente com o cônjuge & 79 & 42,2 & 322 & 48,6 & \\
\hline Com outros de sua geração (com ou sem cônjuge) & 19 & 10,2 & 60 & 9,0 & \\
\hline Com filhos (com ou sem cônjuge) & 43 & 23,0 & 133 & 20,1 & \\
\hline Com netos (com ou sem cônjuge) & 9 & 4,8 & 27 & 4,1 & \\
\hline Outros arranjos & 3 & 1,6 & 19 & 2,9 & \\
\hline \multicolumn{6}{|l|}{ Atividade profissional } \\
\hline Dona de casa & 92 & 49,5 & 246 & 37,1 & \multirow{9}{*}{$<0,001$} \\
\hline Empregada doméstica & 2 & 1,1 & 6 & 0,9 & \\
\hline Trabalhador braçal & 2 & 1,1 & 20 & 3,0 & \\
\hline Trabalhador rural & 29 & 15,6 & 186 & 27,9 & \\
\hline Profissional liberal & - & - & 14 & 2,1 & \\
\hline Agricultor & 3 & 1,6 & 17 & 2,6 & \\
\hline Empresário & - & - & 2 & 0,3 & \\
\hline Outro & 9 & 4,8 & 50 & 7,5 & \\
\hline Não exerce & 49 & 26,3 & 124 & 18,6 & \\
\hline
\end{tabular}

Houve maior proporção de mulheres idosas com indicativo de depressão em relação aos homens $\left(x^{2}=26,064 ; p<0,001\right)$, Tabela 1. Quanto à faixa etária, em ambos os grupos, o maior percentual tinha entre 60 -70 anos, contudo, destaca-se a maior proporção de idosos com 80 anos ou mais com indicativo de depressão em relação aos que não apresentaram o referido indicativo $\left(x^{2}=26,064 ; p=0,034\right)$, Tabela 1.

Obteve maior percentual de idosos que residia somente com o cônjuge tanto para aqueles com indicativo de depressão (42,2\%) como para os sem indicativo (48,6\%), Tabela 1. Na comparação entre os grupos, não houve associação estatisticamente significativa entre o indicativo de depressão e o arranjo de moradia $\left(x^{2}=3,834 ; p=0,573\right)$, Tabela 1.

Prevaleceram os idosos que exerciam suas atividades laborais como donas de casa, com maior proporção entre os com indicativo de depressão, em relação aos que não apresentaram este indicativo $\left(x^{2}=31,912, \quad p<0,001\right)$, Tabela 1.

As atividades de passatempo realizadas pelos idosos, de acordo com a presença de indicativo de depressão, estão apresentadas na Tabela 2. 
Tabela 2: Atividades de passatempo realizadas pelos idosos com e sem indicativo de depressão. Uberaba, MG, 2011.

\begin{tabular}{|c|c|c|c|c|c|c|}
\hline \multirow{2}{*}{\multicolumn{2}{|c|}{ Atividades de passatempo }} & \multicolumn{4}{|c|}{ Depressão } & \multirow[t]{3}{*}{$p$} \\
\hline & & \multicolumn{2}{|c|}{ Sim } & \multicolumn{2}{|c|}{ Não } & \\
\hline & & $\mathbf{N}$ & $\%$ & $\mathbf{N}$ & $\%$ & \\
\hline \multirow[t]{2}{*}{ Assistir televisão } & Sim & 142 & 75,9 & 548 & 82,7 & \multirow{2}{*}{0,038} \\
\hline & Não & 45 & 24,1 & 115 & 17,3 & \\
\hline \multirow[t]{2}{*}{ Ouvir rádio } & Sim & 99 & 52,9 & 449 & 67,7 & \multirow{2}{*}{$<0,001$} \\
\hline & Não & 88 & 47,1 & 214 & 32,3 & \\
\hline \multirow[t]{2}{*}{ Ler } & Sim & 51 & 27,4 & 266 & 40,2 & \multirow{2}{*}{0,001} \\
\hline & Não & 135 & 72,6 & 396 & 59,8 & \\
\hline \multirow[t]{2}{*}{ Jogar (jogos de salão) } & Sim & 26 & 14,0 & 169 & 25,6 & \multirow{2}{*}{0,001} \\
\hline & Não & 160 & 86,0 & 491 & 74,4 & \\
\hline \multirow[t]{2}{*}{ Fazer atividades manuais } & Sim & 66 & 35,5 & 296 & 44,8 & \multirow{2}{*}{0,024} \\
\hline & Não & 120 & 64,5 & 365 & 55,2 & \\
\hline \multirow[t]{2}{*}{ Dançar } & Sim & 36 & 19,4 & 229 & 34,6 & \multirow{2}{*}{$<0,001$} \\
\hline & Não & 150 & 80,6 & 432 & 65,4 & \\
\hline
\end{tabular}

Em relação às atividades de passatempo, assistir televisão foi a mais mencionada pelos idosos, seguido por ouvir rádio, em ambos os grupos, Tabela 2. Houve maior proporção de idosos sem indicativo de depressão que tinham o hábito de assistir televisão $\left(x^{2}=4,309 ; p=0,038\right)$, ouvir rádio $\left(x^{2}=13,913 ; p<0,001\right)$, ler $\left(x^{2}=10,103 ; p=0,001\right)$, jogar $\left(x^{2}=11,061 ; p=0,001\right)$, realizar atividades manuais $\left(x^{2}=5,126 ; p=0,024\right)$ e dançar $\left(x^{2}=15,784 ; p<0,001\right)$ em relação aos que apresentaram o referido indicativo, Tabela 2.

Os hábitos de vida auto-referidos pelos idosos com e sem indicativo de depressão estão dispostas na Tabela 3.

Tabela 3: Hábitos de vida dos idosos segundo o indicativo ou não de depressão. Uberaba, MG, 2011

\begin{tabular}{|c|c|c|c|c|c|c|}
\hline \multirow{2}{*}{\multicolumn{2}{|c|}{ Hábitos de vida }} & \multicolumn{4}{|c|}{ Depressão } & \multirow{3}{*}{$p$} \\
\hline & & \multicolumn{2}{|c|}{ Sim } & \multicolumn{2}{|c|}{ Não } & \\
\hline & & $\mathbf{N}$ & $\%$ & $\mathbf{N}$ & $\%$ & \\
\hline \multirow{2}{*}{ Pratica atividade física } & Sim & 34 & 18,4 & 191 & 29,0 & \multirow{2}{*}{0,004} \\
\hline & Não & 151 & 81,6 & 467 & 71,0 & \\
\hline \multirow{2}{*}{ Tabagista } & Sim & 40 & 21,5 & 118 & 17,9 & \multirow{2}{*}{0,544} \\
\hline & Não & 146 & 78,5 & 541 & 82,1 & \\
\hline \multirow{6}{*}{ Frequência que ingere bebida alcóolica } & Diariamente & 4 & 2,1 & 37 & 5,6 & \multirow{6}{*}{0,008} \\
\hline & 1 a 3 vezes por semana & 12 & 6,4 & 71 & 10,7 & \\
\hline & 4 a 6 vezes por semana & - & - & 10 & 1,5 & \\
\hline & 1 a 3 vezes por mês & 14 & 7,5 & 68 & 10,3 & \\
\hline & Menos de 1 vez por mês & 10 & 5,3 & 39 & 5,9 & \\
\hline & Nenhuma & 147 & 78,6 & 437 & 66,0 & \\
\hline
\end{tabular}

Os idosos que não praticavam atividade física foi, proporcionalmente, maior entre os que apresentavam indicativo de depressão comparados àqueles sem indicativo $\left(x^{2}=8,369 ; p=0,004\right)$, Tabela 3 .

Quanto ao uso de tabaco, houve maior percentual entre os idosos com indicativo de depressão; porém, sem diferença significativa entre os grupos $\left(x^{2}=1,217\right.$; $p=0,544)$, Tabela 3. Concernente ao uso de álcool, a proporção de idosos com indicativo de depressão que não bebiam foi significativamente superior aos sem indicativo $(p=0,008)$, Tabela 3 .

\section{DISCUSSÃO}

A maior proporção de mulheres com indicativo de depressão comparado aos homens corrobora com pesquisa realizada com idosos da zona rural da China $(p<0,001)^{(10)}$. A associação entre a presença de indicativo de depressão e o sexo feminino pode estar relacionada à ocorrência de alterações hormonais, assim como a 
modificação dos aspectos sociais e emocionais vivenciados pelas mulheres nesta faixa etária(19).

Destaca-se que o cuidado ao idoso que reside na zona rural impõe desafios aos profissionais de saúde, sendo necessárias mudanças no arranjo organizacional dos sistemas de saúde e sociais. Em muitos casos a distância entre as unidades de saúde e a residência do idoso, pode inviabilizar a detecção precoce dos casos de depressão e estabelecimento da terapêutica, agravando a doença.

Além disso, ressalta-se o despreparo dos profissionais de saúde em identificar a presença de sintomas depressivos nesta população ou, ainda, as escassas ações voltadas para a prevenção de agravos em saúde mental de promoção da saúde.

Os serviços de Atenção Primária à Saúde se constituem em espaço privilegiado para aplicação de escala de rastreio de depressão. A aplicação rotineira da escala de depressão geriátrica durante a consulta de enfermagem, favorece a identificação dos possíveis casos de depressão entre os idosos, propiciando o diagnóstico precoce e o estabelecimento da terapêutica adequada. Salienta-se a importância do acompanhamento do idoso, bem como da família, pelo enfermeiro e dos demais membros da equipe de saúde, durante o tratamento.

Em relação à faixa etária, estudo de meta-análise que analisou 32 artigos realizados com idosos nas zonas urbana e rural da China condiz com os achados dessa pesquisa, uma vez que verificaram maior percentual de idosos com indicativo de depressão entre 60 -70 anos ${ }^{(20)}$. Os autores destacam que essa faixa etária coincide com o momento em que os idosos se aposentam. Nesse período é possível que alguns idosos enfrentem dificuldades de adaptação, podendo repercutir na presença de sintomas depressivos. Porém, ressaltam que mais estudos devem ser realizados para confirmação dessa hipótese ${ }^{(20)}$.

A maior proporção de idosos com 80 anos ou mais com indicativo de depressão evidenciada nesta pesquisa, corrobora com inquérito conduzido com idosos da zona rural e urbana de Guadalajara, na Espanha, o qual verificou que a idade igual ou superior a 85 anos esteve associada à presença de sintomas depressivos $(p<0,001)^{(9)}$.

Em relação ao arranjo de moradia, estudo realizado com idosos nas zonas urbana e rural de Quebec, no Canadá, destaca que a prevalência de depressão pode ser menor entre aqueles que são casados e residem com o cônjuge, porém apenas quando existe um bom relacionamento entre eles ${ }^{(5)}$.

Verificou-se que o indicativo de depressão não esteve associado ao arranjo de moradia, contudo, pesquisa realizada com idosos residentes na área rural da China obteve resultados contrários ( $p<0,0001)$ e verificou que morar sozinho estava associado ao maior risco para desenvolver depressão, devido à dificuldades relacionadas ao acesso a serviços de saúde e menor apoio social ${ }^{(10)}$. É possível que o maior percentual de idosos que residem apenas com o cônjuge, seja consequência da saída dos filhos de casa, em busca de estudo e oportunidades de trabalho nas cidades. Ainda assim, foi possível observar durante a coleta de dados que, mesmo nesses casos, as relações familiares se mantêm preservadas.

Em relação às atividades laborais, estudo realizado com idosos em Florianópolis-SC verificou o predomínio de mulheres que realizavam atividades domésticas(2). Esse fato pode ter relação com aspectos culturais do passado em que às mulheres eram atribuídas atividades vinculadas ao lar e à família, sem reconhecimento profissional e direito à aposentadoria (2), o que poderia contribuir para a presença de sintomas depressivos.

Referente às atividades de passatempo, é possível que entre os idosos entrevistados existam casos subdiagnosticados de depressão, os quais não estão em tratamento; o que pode ter contribuído para que se sintam desinteressados em realizar essas atividades. Destaca-se, ainda, que as atividades de lazer são relevantes para o controle ou a redução do estresse, da angústia e da depressão. Além disso, podem promover a interação social, ajudando o idoso a manter uma postura positiva perante as situações de dificuldades ${ }^{(13)}$.

Estudo realizado com idosos, na zona urbana de um município de São Paulo, verificou uma correlação fraca $(p=0,001)$ entre apresentar sintomas depressivos e não participar de atividades sociais, como bailes e festas religiosas, entre outras práticas relacionadas ao lazer ${ }^{(21)}$.

O estímulo para que os idosos realizem atividades que promovam, além da distração, a interação social, propicia o maior bem estar, os mantém ativos, e repercute em melhor qualidade de vida e na redução dos sintomas depressivos. A equipe de saúde deve investigar o indicativo de depressão entre os idosos. Quando houver a confirmação diagnóstica é necessário estabelecer e 
acompanhar o tratamento, visando a manutenção da inserção social e dos laços familiares, uma vez que os sintomas depressivos podem desestimular o idoso a realizar atividades de lazer e favorecer o isolamento, agravando a doença.

Quanto à realização de atividade física, assim como nesse inquérito, estudo realizado na zona urbana de Florianópolis-SC verificou que os idosos sem indicativo de depressão, apresentaram maiores percentuais de prática de atividades físicas ${ }^{(2)}$. Em pesquisa conduzida em uma estância mineral no interior de São Paulo obteve que idosos que praticavam atividade física possuíam menos sintomas depressivos do que aqueles que não praticavam ${ }^{(22)}$. Pessoas com depressão podem apresentar menor interesse ou motivação para desenvolver atividades físicas, culturais e sociais, reduzindo, assim, a execução de atividades da vida diária e tornando-as mais sedentárias em casa e na sociedade(2).

Desse modo, é salutar que as ações governamentais favoreçam a presença de um profissional de educação física que, junto à equipe de saúde, estimule, oriente e acompanhe as atividades para idosos na zona rural, em locais adequados e com segurança. Isso poderá contribuir para o cuidado com a saúde física e mental, além de promover a socialização e integração dos idosos com indicativo de depressão na sociedade.

Referente ao uso de tabaco, divergente dessa pesquisa, estudo de revisão sobre os fatores de risco para desenvolvimento de depressão evidenciou associação entre o hábito de fumar e o indicativo de depressão em idosos $^{(23)}$.

Em relação ao uso de álcool, resultados contrários foram verificados em estudo internacional realizado com idosos, o qual evidenciou relação entre uso de álcool e indicativo de depressão(23).

Ainda que a maior proporção de idosos entrevistados tenha referido não fazer uso de álcool, é imprescindível que a equipe de saúde esteja atenta a esta questão, e estimule por meio de atividades de educação em saúde a realização de hábitos saudáveis de vida.

\section{REFERÊNCIAS}

1. Ministério da Saúde; Departamento de Atenção Básica. Envelhecimento e saúde da pessoa idosa. Brasília (Brasil): Ministério da Saúde; 2007.

\section{CONCLUSÕES}

Os resultados obtidos nessa pesquisa demonstram que o indicativo de depressão esteve associado ao sexo feminino, idade igual ou superior a 80 anos, ser dona de casa, não realizar atividades de passatempo, não realizar atividade física e ao uso de álcool.

Destaca-se que devido ao delineamento transversal do estudo não foi possível estabelecer a relação de causalidade entre as variáveis estudadas e a presença de indicativo de depressão entre os idosos residentes na zona rural. Entretanto, ressalta-se que esses resultados podem subsidiar ações de saúde voltadas de modo particular à mulher idosa, àqueles com idade mais avançada, considerando ainda as atividades de passatempo e os hábitos de vida desses idosos.

As equipes de saúde da família representam papel relevante no desempenho de ações de saúde voltadas à população residente na zona rural. Para tanto, os profissionais devem ser preparados e qualificados para desenvolverem tais estratégias, tendo em vista às especificidades culturais, socioeconômicas, além das barreiras geográficas existentes nessas localidades, ainda mais acentuadas para essa clientela.

Ademais, destaca-se a importância de aprofundar o conhecimento sobre a temática da depressão, identificando os possíveis fatores causais, realizando o diagnóstico precoce, para assim estabelecer junto a equipe à terapêutica adequada.

O enfermeiro pode desenvolver ações de educação em saúde a fim de sensibilizar os idosos quanto a importância dos hábitos saudáveis de vida, incluindo a prática de atividade física, atividades de lazer e diminuição e/ou cessação do tabagismo e do uso de álcool. Nessa perspectiva, locais estratégicos existentes na comunidade devem ser valorizados como espaços a serem utilizados nessas atividades, favorecendo ainda a interação social e ampliando a rede de apoio entre os idosos.

\section{APOIO FINANCEIRO: CNPq e FUNEPU.}

2. Benedetti TRB, Borges LJ, Petroski EL, Gonçalves LHT. Atividade física e estado de saúde mental de idosos. Rev Saude Publica. 2008;42(2):302-7.

3. Tier CG, Lunardi VL, Santos SSC. Cuidado ao idoso deprimido e institucionalizado à luz da complexidade. Rev. Eletr. Enf. 
[Internet]. 2008 [cited 2011 out 10];10(2):530-6. Available from: http://www.fen.ufg.br/revista/v10/n2/v10n2a24.htm

4. Instituto Brasileiro de Geografia e Estatística. Rio de Janeiro: Ministério do Planejamento, Orçamento e Gestão (BR). Síntese de indicadores sociais: uma análise das condições de vida da população brasileira 2010.

5. Tahiri SM, Zunzunegui MV, Préville M, Dubé M. Social relationships and depression among people 65 years and over living in rural and urban areas of Quebec. Int J Geriatr Psychiatry. 2009;24(11):1226-36.

6. Alcântara LR, Lopes MJM. Estrutura dos serviços e consumos em saúde por idosos em um contexto rural do sul do Brasil. REDES. 2012;17(1):94-114.

7. Freitas MC, Queiroz TA, Sousa JAV. O significado da velhice e da experiência de envelhecer para idosos. Rev Esc Enferm USP. 2010;44(2):407-12.

8. Morais EP, Rodrigues RAP, Gerhardt TE. Os idosos mais velhos no meio rural: realidade de vida no interior gaúcho. Texto Contexto Enferm. 2008;17(2):374-83.

9. Torija JRU, Mayor JMF, Salazar MPG, Buisán LT, Fernández RMT. Sintomas depresivos en personas mayors. Prevalencia y factores asociados. Gac Sanit. 2007;21(1):37-42.

10. Gao S, Jin Y, Unverzagt FW, Liang C, Hall KS, Ma F, et al. Correlates of depressive symptoms in rural elderly chinese. Int J Geriatr Psychiatry. 2009;24:1358-66.

11. Rocha SV, Almeida MMG, Araújo TM, Virtuoso Júnior JS. Atividade física no lazer e transtornos mentais comuns entre idosos residentes em um município do nordeste do Brasil. J Bras Psiquiatr. 2011;60(2):80-5.

12. Araújo J, Ramos E, Lopes C. Estilos de vida em percepção do estado de saúde em idosos portugueses de zonas rural e urbana. Acta Med Port. 2011;24(S2):79-88.

13. Navarro FM, Rabelo JF, Faria ST, Lopes MCL, Marcon SS. Percepção dos idosos sobre a prática e a importância de atividades físicas em suas vidas. Rev Gaucha Enferm. 2008;29(4):596-603.

14. Datasus [Internet]. Brasília: Ministério da Saúde (BR) [cited 2011 nov 17]. Departamento de Informática do SUS - DATASUS. Available from:

http://tabnet.datasus.gov.br/cgi/deftohtm.exe?ibge/cnv/popuf. def

15. Bertolucci PHF, Brucki SMD, Campacci SR, Juliano Y. O miniexame do estado mental em uma populaçäo geral: impacto da escolaridade. Arq Neuropsiquiatr. 1994;52(1):1-7.

16. Ramos LR. Growing old in São Paulo, Brazil. Assessment of health status and family support of the elderly of different socio-economic strata living in the community [thesis]. London: London School of Hygiene and Tropical; 1987.

17. Frank MH, Rodrigues NL. Depressão, ansiedade, outros distúrbios afetivos e suicídio. In: Freitas EV, Py EV, Neri AL. Tratado de geriatria e gerontologia. Rio de Janeiro: Guanabara Koogan; 2006, p. 376-87.

18. Almeida OP, Almeida SA. Confiabilidade da versão brasileira da Escala de Depressão em Geriatria (GDS) versão reduzida. Arq Neuropsiquiatr. 1999;57(2-B):421-6.

19. Polisseni AF, Araújo DAC, Polisseni F, Mourão Junior CA, Polisseni J, Fernandes ES, et al. Depressão e ansiedade em mulheres climatéricas: fatores associados. Rev Bras Ginecol Obstet. 2009;31(1):28-34.

Zhang $L, X u Y$, Nie $H$, Zhang $Y$, Wu Y. The prevalence of depressive symptoms among the older in China: a meta-analysis. Int J Geriatr Psychiatry. 2012;27:900-6.

Baptista MN, Morais PR, Rodrigues T, Silva JAC. Correlação entre sintomatologia depressiva e prática de atividades sociais em idosos. Avaliação psicologica. 2006;5(1):77-85.

20. Domingues PC, Neri AL. Atividade física habitual, sintomas depressivos e doenças auto-relatadas em idosos da comunidade. Revista brasileira de atividade física \& saúde. 2009;14(3):164-73.

23. Vink D, Aartsen MJ, Schoevers RA. Risk factors for anxiety and depression in the elderly: a review. J Affect Disord. 2008;106(1-2):29-44.

Artigo recebido em 22/12/2011. Aprovado para publicação em 13/08/2012. Artigo publicado em 31/03/2013. 